A phenomenon that is present in almost every shot is highlighted. It manifests itself in the muzzle discharge as a certain amount of free carbon. The thermochemical reaction of Boudouard-Bell (disproportionation of carbon monoxide) was determined, which explains the formation of free carbon in the gunpowder gases during the firing process. A feature of this reaction is the formation of a condensed phase of carbon during the firing process after the gasification of the gunpowder charge.

The reason is revealed that does not allow describing the formation of free carbon during firing on the basis of existing models of internal ballistics processes. It is the lack of taking into account the temperature distribution of the gunpowder gases along the length of the gun barrel and its change. A mathematical model is proposed that makes it possible to estimate the temperature distribution during the shot.

A method has been developed for solving the problem of internal ballistics with the ability to determine the temperature of gunpowder gases along the length of the gun barrel at different times and at different positions of the projectile in the barrel. The original model is built using generally accepted assumptions. Modeling results can only be estimated. For this reason, the method is based on simple calculations, which makes it possible not to involve highpower computing equipment.

The modeling of the temperature distribution of gunpowder gases in the space of the gun barrel between the charging ball and the moving projectile in the model system is carried out. The possibility of changing the length of the zone of the Boudouard-Bell reaction (the zone of formation of free carbon) depending on the initial data is shown. The use of a fresh gunpowder charge and a degraded one is simulated. Full and reduced charges are considered. The simulation results showed the reason for the possibility of initiating a secondary muzzle discharge flash both from the front side and from the side of the muzzle brake

Keywords: gun, gunpowder gases, temperature distribution, disproportionation reaction, free carbon, muzzle flash

\section{DEVELOPMENT OF THE MODEL AND THE METHOD FOR DETERMINING THE INFLUENCE OF THE TEMPERATURE OF GUNPOWDER GASES IN THE GUN BARREL FOR EXPLAINING VISUALIZE OF FREE CARBON AT SHOT}

\author{
O lexander Brunetkin \\ Corresponding author
}

Doctor of Technical Sciences, Professor*

E-mail: brunetkin@opu.ua

Maks y Maks y mov

Doctor of Technical Sciences, Professor, Chief Researcher Scientific Center**

VIadimir Brunetkin

Postgraduate Student*

Oleksii Maksymov*

Yevhenii Dobrynin Researcher**

Vitali i Kuzmenko Researcher**

Pavlo Gultsov Postgraduate Student*

*Department of Computer Automation Technologies

Odessa Polytechnic State University

Shevchenko ave., 1, Odesa, Ukraine, 65044

** Institute of Naval Forces

National University "Odessa Maritime Academy»

Didrichsona str., 8, Odessa, Ukraine, 65029
Received date 29.06.2021

Accepted date 23.08.2021 Published date 31.08.2021
How to Cite: Brunetkin, O., Maksymov, M., Brunetkin, V., Maksymov, O., Dobrynin, Y., Kuzmenko, V., Gultsov, P. (2021). Evelopment of the model and the method for determining the influence of the temperature of gunpowder gases in the gun barrel for explaining visualize of free carbon at shot. Eastern-European Journal of Enterprise Technologies, 4 (1 (112)), $41-53$. doi: https://doi.org/10.15587/1729-4061.2021.239150

\section{Introduction}

From a technical point of view, the performance of the task during practical firing with shot artillery installations depends on two components: the state of the charge for firing, equipped at the position, and the state of the receiver system. The authors' experience of practical shooting shows that the possibility of carrying out full-scale routine checks in accordance with the existing organizational and technical complex of measures in the process of practical shooting does not always exist.

The initial velocity of the projectile and the range of its deviation during practical firing with charged charges, which are conditionally assigned to one batch, must lie within acceptable, controlled limits. It is understood that this is ensured by the coincidence of the year of assembly and the number (code) of the equipment factory. But even if they coincide, the quality of the individual charges to be completed differ significantly from 
each other. Moreover, such data may be absent altogether, especially under conditions of intensive practical shooting in the event of a shortage of charges and with their expired storage.

All other things being equal, the height of the barrel also affects the changes in the ballistic characteristics of the shot. The caliber of the barrel increases and, accordingly, the volume, where the first pyrodynamic period takes place. The cost of a barrel of large caliber artillery mounts is estimated at 30-35\% of the cost of an installation. Operation of a well that has reached the standard wear makes it impossible to restore it in the field.

Controlling the state of the gunpowder charge and the barrel system is important as a means of ensuring the ballistic characteristics of the projectile. At the moment, the corresponding characteristics can be determined using ballistic radar stations. But, on the one hand, this approach is expensive. On the other hand, during the operation of such stations, the adjacent side immediately determines them with the available means, since these means belong to the category of active ones. At the same time, there are methods for assessing barrel wear and the state of charges based on acoustic fields recorded at large distances. Acoustic analysis methods are simple, economical, do not require expensive equipment, and are applicable in the field. They belong to the category of passive and can't be fixed by the adjacent side. The assessment method is based on the measurement of muzzle and ballistic wave indicators and the analysis of sound waves that make up the shot. The analysis is based on the time, frequency and cumulative features of these waves.

As criteria for assessing the quality of the information received on the basis of acoustic methods, let's use the indicators of the reliability and probability of an error of the second kind. The results obtained were 0.94/0.04, respectively, which does not correspond to the required characteristic of 0.99/0.01 for the practical application of such systems when analyzing the state of the charge - charging chamber - barrel system. Achievement of the necessary indicators can be ensured by taking into account additional information signs of a shot that characterize the system charge - charging chamber - barrel. In their capacity, a visual manifestation of a shot in the form of a muzzle discharge and a muzzle flash can be used.

Comparison of various muzzle emissions makes it possible to single out the presence of free carbon as one of the information indicators. Free carbon may not take up all of the emissions. This allows to conclude about its formation in the barrel at the previous stage of the shot and actualizes the search for a model for the formation of free carbon. It is necessary to further develop the mathematical model of the physicochemical processes accompanying the shot and methods for its solution. This model can serve as a prerequisite for the development of systems for diagnosing the state of the barrel - charge projectile system based on an integral indicator. It can assess: the technical condition of the gun barrel; the effect of the gunpowder charge on the initial velocity of the projectile. The listed characteristics should be taken into account when solving computational and informational problems of preparing data for firing using an ACS with artillery subunits fire.

\section{Literature review and problem statement}

If the presence of free carbon is recorded in the muzzle discharge, and the known models do not reflect this, then the purpose of analyzing the literature data is to find grounds for creating such a model. The formation of free carbon is considered as a fact confirming the need to improve existing models.
Mathematical models (MM) used to determine the parameters of the shot differ mainly in the degree of detail in describing these processes. So, in its simplest form, it can be a model in a concentrated setting and a classical analytical [1] solution. Despite the development of computer technology, due to its relative simplicity, clarity and satisfactory results, the analytical solution remains in demand at the present time [2]. The desire to obtain more accurate results with fewer assumptions led to the improvement of methods for solving systems of ordinary differential equations based on numerical methods [3]. With the development of numerical methods and the availability of computing resources, distributed models appeared [4], as well as models that take into account the possibility of a two-phase flow of gunpowder gases (PG) [5]. In the latter case, particles of burning gunpowder are considered as the condensed phase. A consequence of the complexity of the processes accompanying the shot is the limited accuracy of their mathematical description. As a result, the models contain empirical coefficients that make it possible to match the calculated parameters of the processes with the corresponding values in experimental studies and practical shooting. The above examples of improving MM and methods for their solution [3-5] made it possible to reduce the influence of empirical coefficients, but did not eliminate it.

Many works do not consider the issue of PG temperature distribution along the barrel length. This excludes the possibility of taking into account its influence on the processes of internal ballistics. In the listed works, only in [5] this issue was touched upon and the identification of an effect that does not fit into the classical concept of the temperature distribution of combustion products was immediately noted. It manifests itself in a significant increase in temperature at the bottom of the channel and the bottom of the projectile. In [5], this phenomenon is explained as the Mach effect. It consists in increasing the temperature in the first portions of the formed gases due to the compression of the next formed gases. This approach can be taken into consideration for the PG at the bottom of the projectile. But at the bottom of the channel, PGs are mostly formed at the last moments of gunpowder transformation, at a lower and ever decreasing pressure. This does not explain the rise in temperature at the bottom of the barrel.

Internal ballistics processes are difficult to study instrumental. But their consequences should be reflected in external manifestations, in particular in the nature of the formation and course of the muzzle flash. An attempt to use such a relationship is realized in a number of works. For example, in [6], the main task is to study the PG flow in the presence of a muzzle brake compensator. As the initial data for its solution, the results of the numerical solution of the internal ballistics problem are used, taking into account the chemical kinetics of the reactions of the combustion of gunpowder in the barrel and PG in a muzzle flash. The phenomenon of a secondary flash of PGs has been noted. The gases $\mathrm{CO}$ and $\mathrm{H}_{2}$ are indicated as the main combustible component. But the frame-by-frame study of the video sequence of the development of the muzzle flash of some guns, as well as data from a number of works, indicate the need to make adjustments to the models of the processes under consideration. So, for example, in the photographs in Fig. 1, 2, taken from different angles, a shot of $152 \mathrm{~mm} 2 \mathrm{~A} 36$ shot at the initial moment of muzzle discharge shows some volumes of black matter. These can be identified as free carbon emissions. Its presence in the muzzle discharge is also noted in [7]. Photographs of $155 \mathrm{~mm}$ M109 self-propelled guns (Paladin, USA) (Fig. 3) also confirm the presence of black matter in the muzzle discharge. 


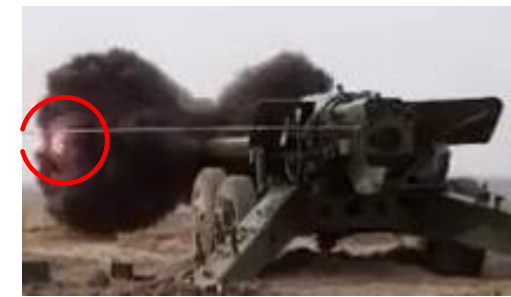

$a$

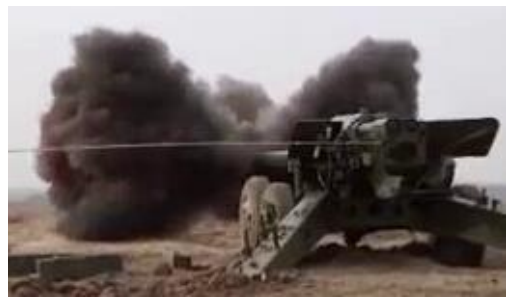

$b$

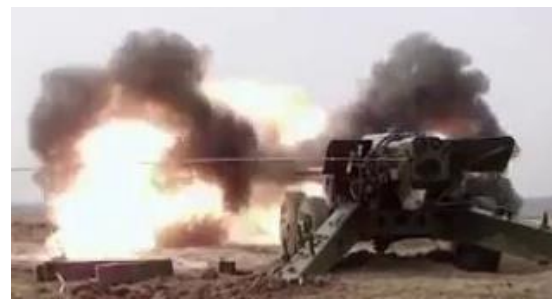

C

Fig. 1. Stages of development of muzzle discharge of a $152 \mathrm{~mm}$ shot2A36 shot taken from ground level: $a$ - initiation of a flash; $b$ - development of muzzle discharge; $c$ - muzzle discharge flash

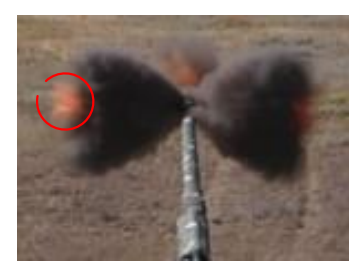

$a$

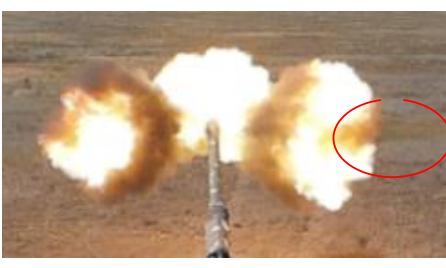

$b$

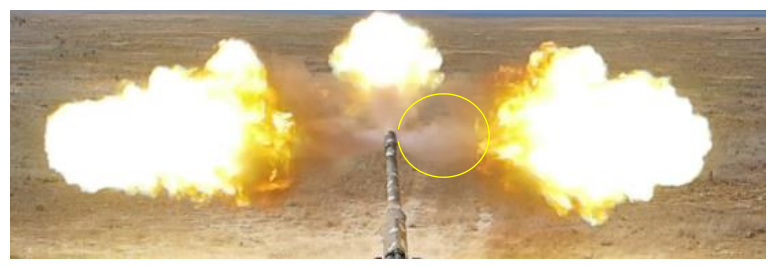

c

Fig. 2. Stages of development of the muzzle discharge of a $152 \mathrm{~mm}$ shot $2 \mathrm{~A} 36$ shot taken from the elevation point: $a$ - initiation of a muzzle flash; $b$ - development of muzzle flash; $c$ - the end of the muzzle flash (author's photos)

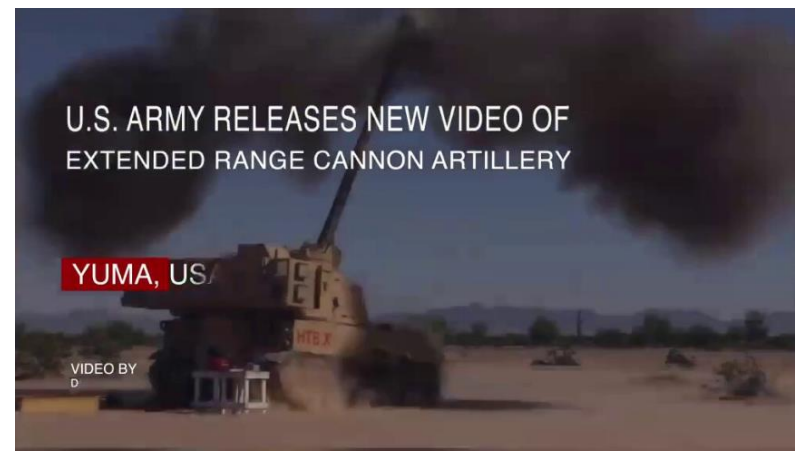

$a$

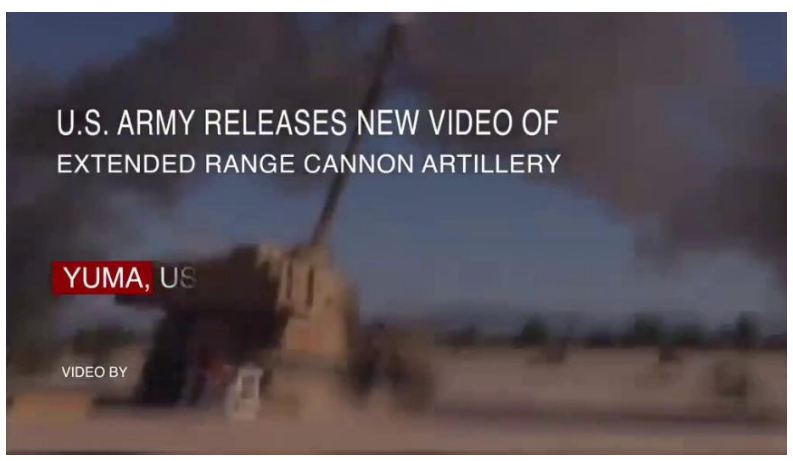

$b$

Fig. 3. Development stages of muzzle discharge of $155 \mathrm{~mm}$ self-propelled shot M109 (Paladin). The muzzle flash did not occur. Polygon YUMA. The official Twitter of the US Army: $a$ - the moment of the maximum emission of free carbon; $b$ - scattering muzzle discharge without its secondary flash

In the composition of $\mathrm{NC}$ gunpowder, there is not enough oxygen for oxidation according to the maximum valence of all chemical elements included in it. The deficit can be $20 \%$. In this case, when solving problems of internal ballistics, taking into account the chemical kinetics of the combustion processes of gunpowder, the conditions for the possible formation of a condensed phase in the form of free carbon are not distinguished.

The presence of free carbon in the muzzle discharge is paid attention to by specialists investigating the problems of determining the type and location of the weapon by the muzzle flash [8]. In addition, the possibility of determining the type of projectile based on the registration of the effects of the external manifestation of the shot is being considered [9]. An attempt to use phenomenological data on the composition, temperature, and muzzle flash energy emphasizes the understanding of the importance of such parameters. But significant deviations when comparing experimental data and calculation results indicate the absence of appropriate adequate models for their determination.

There is no explanation in the description of some of the processes occurring in the muzzle flash. Thus, in [6], the secondary flash is explained by the ignition of the PG components in the form of $\mathrm{CO}$ and $\mathrm{H}_{2}$ in the air. Muzzle blast is con- sidered as the reason for additional heating of the mixture. Due to a certain area of its boundary, one could expect the ignition of the mixture over a certain surface. At the same time, the photos in (Fig. 1, $a$ ) and (Fig. 2, $a$ ) allow to see the point of ignition at the leading edge in the center (highlighted in a red circle). This effect is known and noted even in the educational literature [10] as a result of field tests, but the explanation is not given in it either.

It is of interest to study the effect of barrel wear and gunpowder degradation on the parameters of the shot process.

The works [11,12] investigate the effect of barrel wear and gunpowder degradation on the parameters of the shot process. The results are obtained by numerical studies based on known models. At the same time, in practice, the models used do not take into account the physicochemical phenomena of the shot, which determine the formation of free carbon.

The results obtained are not compared with the effects in the external manifestation of the shot. So in [11] only the shape of the hole in the target is considered. Its elliptical shape is explained by an increase in the angle of attack of the projectile due to a decrease in its rotation speed when the barrel is worn. But in practical cases, the speed of rotation of the projectile can be reduced even at a lower speed of the projectile at the exit from the barrel due to degradation of the 
gunpowder. In [12], the process parameters calculated taking into account barrel wear and gunpowder degradation are not associated with the effects of the external manifestation of the shot, which can be controlled.

The reliability of the calculation results can be influenced to a greater extent not even by the accuracy of the initial data, but by the completeness of accounting for the effects in the MM of the process under study. Especially if the result of the manifestation of these effects can be controlled by available means. The presence of free carbon in the muzzle discharge indicates a chemical reaction that promotes its formation. The reaction rate, the position of its equilibrium point depends on the temperature of the reaction zone. The different localization of free carbon along the length of the discharge indicates the variability of the conditions of its formation, first of all, the temperature. But most of the existing models describing the physicochemical phenomena during the firing process do not take into account the possibility of any chemical reaction, except for the transformation of gunpowder. Accordingly, the conditions contributing to it are not analyzed.

The identification and description of the effect leading to the formation of free carbon will contribute to the solution of the applied problem. It consists in the need, with each shot, to determine the effectiveness of the state of the system charge - charging chamber - barrel in the case of practical firing with limited time and the impossibility of using diagnostic tools.

The best way to obtain the necessary operational data for the formation of the current shot is the information obtained from the previous shot. In the field, this can only be done by analyzing the corresponding perturbations applied to the existing physical fields. The corresponding data can be obtained on the basis of indirect measurements of acoustic fields and visual external manifestations of the shot.

The solution of such an applied problem will make it possible, in the field of practical shooting, with some organizational and technical limitations, to improve the operational diagnostics of artillery shots in the real time range. It is possible to obtain the desired result only as a result of the search for scientifically grounded models and methods to ensure the specified effectiveness of practical shooting in the case of many restrictions that are present in real conditions.

\section{The aim and objectives of research}

The aim of research is to develop a mathematical model that takes into account the possibility of free carbon formation during the firing process, and a method for determining the distribution of the PG temperature along the barrel length to identify modes that contribute to the formation of free carbon during firing.

To achieve the aim, the following objectives were set:

- to determine the process capable of providing the formation of free carbon in the gunpowder gases in the process of their expansion in the process of firing;

- to develop a method for determining the change in the temperature of gunpowder gases along the length of the barrel in the process of firing;

- to simulate the temperature distribution in the space of the barrel between the charging ball and the moving projectile with the determination of the upper estimate of the amount of released free carbon.

\section{Materials and methods of research}

The widespread use of models in a concentrated formulation to describe the processes of internal ballistics is due to the accuracy of the results obtained on their basis with the relative simplicity of calculations. But this is achieved by introducing empirical coefficients. Their presence indicates the presence of a number of processes and phenomena that are not taken into account in the models used.

Such models are based on the Resal equation [1], which is the expression of the first law of thermodynamics for the adiabatic process of expansion of PGs. This equation connects the PG pressure with the projectile velocity at a given time, depending on the gas flow of the burning gunpowder charge and the barrel geometry. The amount of gas flow, in turn, depends on the PG pressure at the current time and the shape of the gunpowder grain. Another feature of the Resal equation is the use of the force of gunpowder as an energy characteristic for the generated PGs.

In the study, improved models were used that simulate the processes of internal ballistics of barrel systems. Models of chemical kinetics [20] of the formation of PGs [17], models of the processes of their expansion [1] when the projectile moves in the barrel [2] were used as initial models for obtaining modified versions. During the movement of the projectile along the barrel, the maximum value of the PG pressure can reach values of $\sim 300-500 \mathrm{MPa}$. This pressure, as well as the high temperature, justify the assumption of the equilibrium state (or close to it) of chemical reactions between the PG components. The determination of the composition of the PG is based on the solution of the system, which includes the equations: the law of mass action, the law of conservation of matter, Dalton's law [17].

A distinctive feature is the form of the equations of the law of mass action. The equations for the formation of possible PG components from elementary chemicals are used as a basis for their recording [13]. For example:

$$
\begin{aligned}
& \mathrm{H}_{2} \mathrm{O} \rightleftarrows 2 \cdot \mathrm{H}+\mathrm{O} ; \\
& \mathrm{CO}_{2} \rightleftarrows \mathrm{C}+2 \cdot \mathrm{O} .
\end{aligned}
$$

This form of notation is universal. It allows to obtain a closed system of equations based on a list of substances that may be present in a mixture. In the accepted models describing the process of a shot, for $\mathrm{NC}$ gunpowder, the PG composition is determined based on the following list of its components:

$$
\mathrm{H}_{2} \mathrm{O}, \mathrm{CO}_{2}, \mathrm{CO}, \mathrm{H}_{2}, \mathrm{CH}_{4}, \mathrm{~N}_{2} \text {. }
$$

As an improvement to the generally accepted models for displaying the possibility of free carbon formation, the list of components (2) was supplemented with another one and designated by the symbol $\mathrm{F}_{\mathrm{C}}$ (free carbon, fine condensed phase of free carbon). A method for calculating the thermodynamic parameters of a mixture of gaseous products during the formation of a condensed phase is given in [13].

For substances (2), the equilibrium of chemical reactions in the PG gas phase is a generally accepted assumption and is determined by their high rate. There is no information in the literature on the nature of the reaction that could explain the formation of the condensed phase of carbon in the barrel during the firing process. In this case, the amount of the condensed phase calculated on the basis of the equilibrium equations of chemical kinetics can be considered 
only as a necessary factor (condition). As a reaction that can explain this process, disproportionation of carbon monoxide (Boudouard-Bell reaction) is considered [14-17].

$$
2 \cdot \mathrm{CO} \uparrow \rightarrow \mathrm{C} \downarrow+\mathrm{CO}_{2} \uparrow+(Q) .
$$

This reaction is characterized by a temperature range limited from above within which it can be carried out.

Most of the models describing the processes of internal ballistics of barrel systems are based on the assumption of uniformity of pressure and temperature values in the projectile space [4]. This is due to the use of models in a concentrated setting. At the same time, the examples of muzzle blows given above indicate the heterogeneity of free carbon release along the length of the barrel. Consequently, the PG temperature must also be inhomogeneous. In [5], when performing some numerical calculations, an effect was revealed that does not fit into the classical concept of the temperature distribution of gunpowder decomposition products. It manifests itself in a significant increase in temperature at the bottom of the channel and the bottom of the projectile. In [5], this phenomenon is explained as the Mach effect. This approach can be taken into consideration for the PG at the bottom of the projectile. It should be noted that in the literature, the manifestation of the Mach effect is considered during the combustion of condensed substances and gaseous mixtures in a closed (constant) volume. In this case, the pressure of the combustion products increases, which leads to the compression of the previous portions and their heating. Compression occurs due to the work performed by the newly formed portions of combustion products. In this case, their temperature decreases in relation to the combustion temperature.

A similar situation develops at the initial stage of the transformation of gunpowder. Although the volume of space behind the projectile does not remain constant, its magnitude changes relatively slowly compared to the gas flow rate. The PG pressure increases, which leads to their heating at the bottom of the projectile. In this case, the newly formed PGs perform the work of expansion at a high value of pressure. This leads to a significant cooling of the newly formed gases in relation to the combustion temperature.

Subsequently, with an increase in the speed of the projectile and, accordingly, the rate of increase in the volume of space behind the projectile, the magnitude of the PG pressure begins to decrease. In this case, conditions are created for a kind of manifestation of the Mach effect. The formation of PGs continues and their newly formed portions continue to perform the expansion work. This also leads to a decrease in their temperature compared to the temperature of formation. But the work of expansion is performed at a lower value of the PG pressure. Accordingly, the temperature value of the newly formed gases changes to a lesser extent than that of the PG at the previous stages of the firing process. This can explain the increase in PG pressure at the bottom of the channel (barrel).

Taking into account the temperature distribution along the barrel length to determine the possibility of the disproportionation reaction requires considering the problem of internal ballistics in a distributed formulation and for its solution leads to the use of complex numerical methods. At the initial stage of the study of the possibility of manifestation of the phenomenon of disproportionation of carbon monoxide in the process of a shot, such an approach seems to be irrational. This entails the need to improve not only the process model, but also the solution method. The analogue of the finite ele- ment method is taken as a basis. One of the areas of improvement concerns the selection of the defining characteristic of the finite element. In some cases, the unchanged volume of the finite element is taken as the defining characteristic. In this case, its mass is taken as the defining characteristic. The masses of all elements are taken to be constant.

\section{Results of the study of the expansion of gunpowder gases in the process of firing}

5. 1. Determination of the process capable of providing the formation of free carbon in the PG during the shot

The initial parameters for the considered process of expansion of gunpowder gases are the following data:

- possible composition of $\mathrm{PG}$ in the form of the following substances: $\mathrm{H}_{2} \mathrm{O}, \mathrm{CO}_{2}, \mathrm{CO}, \mathrm{H}_{2}, \mathrm{CH}_{4}, \mathrm{~N}_{2}$, including finely dispersed condensed phase of carbon FC (free carbon);

- degradation formula for plastic blasting explosives in the form $[18,19]$ :

$$
\begin{aligned}
& \mathrm{C}_{6} \mathrm{H}_{7.7} \mathrm{~N}_{2.3} \mathrm{O}_{9.6} \rightarrow \mathrm{C}_{5.95} \mathrm{H}_{7.99} \mathrm{~N}_{1.9} \mathrm{O}_{8.76}+ \\
& + \text { DPAnitro derivatives }+0.001 \mathrm{~N}_{2}+ \\
& +0.024 \mathrm{~N}_{2} \mathrm{O}+0.012 \mathrm{CO}+0.012 \mathrm{H}_{2} \mathrm{O},
\end{aligned}
$$

where DPA - diphenylamine;

- the following parameters are accepted: caliber $-d$, $\mathrm{m}$; barrel cross-sectional area $-S, \mathrm{~m}^{2}$; free volume of the charging chamber (excluding the volume of gunpowder) $-W_{C C}, \mathrm{~m}^{3}$; the length of the free volume of the charging chamber $-x_{C C}, \mathrm{~m}$; projectile mass - $M$, kg; gunpowder mass full/reduced charge $-m, \mathrm{~kg}$; corresponding projectile velocity $V$ at the muzzle $-\mathrm{m} / \mathrm{s}$; gunpowder force $-f, \mathrm{~J} / \mathrm{kg}$; adiabatic exponent $-\gamma$; the length of the projectile space in the barrels as the sum of the lengths of the rifled part of the barrel and $x_{C C}-\mathrm{m}$;

Accepted limitations and assumptions:

- in the PG gas phase, the molar mass is constant;

- the linear law of combustion of the gunpowder charge $u_{1}=u_{0} \cdot P$ and the constant area of its combustion $F$. Here $u_{0}$ - combustion rate of the gunpowder, $P$ - pressure at which the gunpowder is burned at a given moment.

Based on the composition of the NC gunpowder (Table 1), the gross formula of the gunpowder charge was obtained, normalized to one carbon atom [20]:

$$
\mathrm{C}_{1} \mathrm{H}_{1.44} \mathrm{~N}_{0.37} \mathrm{O}_{1.57}
$$

which corresponds to the PG gross formula [18]:

$$
\mathrm{C}_{6} \mathrm{H}_{8.64} \mathrm{~N}_{2.22} \mathrm{O}_{9.42} \text {. }
$$

The specific enthalpy of the gunpowder charge is calculated based on the strength of the gunpowder in accordance with the expression:

$$
I_{s}^{p}=I_{s}^{P G}=\frac{\gamma}{\gamma-1} \cdot f,
$$

where $I_{s}^{p}$ - specific enthalpy of the gunpowder; $I_{s}^{P G}$ - specific enthalpy of the gunpowder gases; $\gamma$ - adiabatic index, and $f$ - force of the gunpowder.

Taking into account (4), (6) and (1), the composition of the PG (2) is determined from the equations of the laws of chemical kinetics [20] (the law of mass action), material balance and the Dalton equation. 
Table 1

The original composition of the charge NC gunpowder

\begin{tabular}{|c|c|c|c|}
\hline Component & Substance & $\%$ & Gross formula \\
\hline Energy base & nitrocellulose & 96.0 & $\mathrm{C}_{6} \mathrm{H}_{7.7} \mathrm{~N}_{2.3} \mathrm{O}_{9.6}$ \\
\hline \multirow{2}{*}{ Plasticizer } & ethanol & 0.5 & $\mathrm{C}_{2} \mathrm{H}_{6} \mathrm{O}$ \\
\cline { 2 - 4 } & diethyl ether & 0.5 & $\mathrm{C}_{4} \mathrm{H}_{10} \mathrm{O}$ \\
\hline $\begin{array}{c}\text { Chemical resistance } \\
\text { stabilizer }\end{array}$ & diphenylamine & 1.0 & $\mathrm{C}_{12} \mathrm{H}_{11} \mathrm{~N}$ \\
\hline Substances-parasites & water (moisture) & 2.0 & $\mathrm{H}_{2} \mathrm{O}$ \\
\hline
\end{tabular}

Mass action law:

$$
\begin{aligned}
& \frac{P_{\mathrm{C}} \cdot P_{\mathrm{O}}}{P_{\mathrm{CO}}}=K_{\mathrm{CO}}(T), \\
& \frac{P_{\mathrm{C}} \cdot P_{\mathrm{O}}^{2}}{P_{\mathrm{CO}_{2}}}=K_{\mathrm{CO}_{2}}(T), \\
& \frac{P_{\mathrm{H}}^{2} \cdot P_{\mathrm{O}}}{P_{\mathrm{H}_{2} \mathrm{O}}}=K_{\mathrm{H}_{2} \mathrm{O}}(T), \\
& \frac{P_{\mathrm{H}}^{2}}{P_{\mathrm{H}_{2}}}=K_{\mathrm{H}_{2}}(T), \\
& \frac{P_{\mathrm{N}}^{2}}{P_{\mathrm{N}_{2}}}=K_{\mathrm{N}_{2}}(T), \\
& \frac{P_{\mathrm{C}} \cdot P_{\mathrm{H}}^{4}}{P_{\mathrm{CH}_{4}}}=K_{\mathrm{CH}_{4}}(T), \\
& \frac{P_{\mathrm{C}}}{P_{F_{C}}}=K_{F_{C}}(T) .
\end{aligned}
$$

Here $K_{\mathrm{CO}}(T), K_{\mathrm{CO}_{2}}(T), K_{\mathrm{H}_{2} \mathrm{O}}(T), K_{\mathrm{H}_{2}}(T), K_{\mathrm{N}_{2}}(T), K_{\mathrm{CH}_{4}}(T)$, $K_{F_{C}}(T)$ - temperature-dependent constants of chemical equilibrium for the reactions of formation of substances of the form (1).

Material balance equations: $\mathrm{H}_{2} \mathrm{O}, \mathrm{CO}_{2}, \mathrm{CO}, \mathrm{H}_{2}, \mathrm{CH}_{4}, \mathrm{~N}_{2}$ :

$$
\begin{aligned}
& \text { for }[\mathrm{C}] \\
& b_{\mathrm{C}} \cdot M_{T}=P_{\mathrm{CO}}+P_{\mathrm{CO}_{2}}+P_{F_{C}}+P_{\mathrm{C}}, \\
& \text { for }[\mathrm{H}] \\
& b_{\mathrm{H}} \cdot M_{T}=2 \cdot P_{\mathrm{H}_{2} \mathrm{O}}+2 \cdot P_{\mathrm{H}_{2}}+4 \cdot P_{\mathrm{CH}_{4}}+P_{\mathrm{H}}, \\
& \text { for }[\mathrm{O}] \\
& b_{\mathrm{O}} \cdot M_{T}=P_{\mathrm{CO}}+2 \cdot P_{\mathrm{CO}_{2}}+P_{\mathrm{H}_{2} \mathrm{O}}+P_{\mathrm{O}}, \\
& \text { for }[\mathrm{N}] \\
& b_{\mathrm{N}} \cdot M_{T}=2 \cdot P_{\mathrm{N}_{2}}+P_{\mathrm{N}} .
\end{aligned}
$$

Here $b_{\mathrm{C}}, b_{\mathrm{H}}, b_{\mathrm{O}}, b_{\mathrm{N}}-$ the number of atoms, respectively, of carbon, hydrogen, oxygen, nitrogen in the gross formula of gunpowder (4); $M_{T}$ - number of moles of gunpowder (4), which ensures the numerical equality of the pressures of the NG components to their concentrations.

Dalton's equation:

$$
P=P_{\mathrm{CO}}+P_{\mathrm{CO}_{2}}+P_{\mathrm{H}_{2} \mathrm{O}}+P_{\mathrm{H}_{2}}+P_{\mathrm{N}_{2}}+P_{\mathrm{C}}+P_{\mathrm{H}}+P_{\mathrm{O}}+P_{\mathrm{N}}
$$

Here $P$ - total pressure of the components of the gunpowder gases. One should distinguish between the PG in (9) and in (7), (8). $P_{C}$ is the partial pressure of carbon vapor.
$P_{C}$ denotes the concentration of the condensed phase of carbon. The designation of this quantity in the form of pressure is due to the adopted scheme of designations and calculations, in which the concentrations of substances are numerically equal to their partial pressures.

According to the combustion law $u_{1}=u_{0} \cdot P$ and the combustion area $F$, the combustion and expansion times of the PG are determined at the next calculation step. According to this:

$$
m_{i}=\left(u_{0} \cdot P\right) \cdot F \cdot t_{i} \Rightarrow t_{i}=\frac{m_{i}}{\left(u_{0} \cdot F\right) \cdot P} .
$$

The enthalpy $\left(I_{i}^{n}\right)$ of PG is determined as the sum of their enthalpy from the end of the previous step of calculation and introduced with the current part. In accordance with:

$$
I_{i}^{n}=\frac{\gamma}{\gamma-1} \cdot P_{i}^{n} \cdot W_{i}^{n} \Rightarrow P_{i}^{n}=\frac{\gamma-1}{\gamma} \cdot \frac{I_{i}^{n}}{W_{i}^{n}}
$$

the pressure of the PG mixture $\left(P_{i}^{n}\right)$ at the beginning of the calculation step is determined. Here is the volume of the PG element at the calculation step.

The PG expansion and the acceleration of the projectile at the current calculation step occurs during the time $t_{i}$ and is described by the ratio:

$$
\varphi M \frac{d^{2} x}{d t^{2}}=P \cdot S .
$$

Here $M$ - mass of the projectile, $\varphi$ - coefficient taking into account the fictitious value of the mass of the projectile, $x$ - length of the projectile space of the barrel.

The physicochemical processes of PG formation differ from the classical concept of the combustion of gases in an oxidizing atmosphere. The heat transfer process has an insignificant effect on the change in the PG composition and, as a consequence, on the composition of the products obtained. As a result, the ratio for the adiabatic process of gas expansion was used to calculate the value of $P$ :

$$
\left(P_{i}^{n}\right) \cdot\left(W_{i}^{n}\right)^{\gamma}=\left(P_{i}\right) \cdot\left(W_{i}\right)^{\gamma} \Rightarrow P_{i}=\left(P_{i}^{n}\right) \cdot\left(\frac{W_{i}^{n}}{W_{i}}\right)^{\gamma},
$$

where $P_{i}^{n}, W_{i}^{n}$ - values of pressure and volume of the projectile space at the initial moment at the calculation step (corresponding values at the end of the previous calculation step); $P_{i}, W_{i}$ - values of pressure and volume at the current moment of calculation.

Taking into account $W_{i}=S \cdot x_{i}$ and (13), expression (12) can be written as:

$$
\frac{d^{2} x}{d t^{2}}-\frac{r}{(x)^{\gamma}}=0, \text { where } r=\frac{P_{i}^{n} \cdot S \cdot\left(x_{i}^{n}\right)^{\gamma}}{\varphi \cdot M} .
$$

Here $x_{i}^{n}$ - length of the projectile space at the initial moment of the calculation step.

For the analytical solution of (14) to be possible, the second term of the equation is linearized to a point $x_{i}^{n}$ based on the Taylor series. Solution (14) will be written as:

$$
x(t)=\frac{v^{n}}{\sqrt{d_{1}}} \sin \left(\sqrt{d_{1}} \cdot t\right)-\frac{5}{6} x_{i}^{n} \cos \left(\sqrt{d_{1}} \cdot t\right)+\frac{11}{6} x_{i}^{n},
$$

where $d_{1}=\frac{6 r}{5 x_{i}^{n}}$. 
Substituting the value of $t_{i}$, into this expression, the size of the projectile space $x_{i}^{n}$ is determined, and from the derivative of (15):

$$
v=x^{\prime}(t)=v^{n} \cos \left(\sqrt{d_{1}} \cdot t\right)+\frac{5}{6} x_{i}^{n} \sqrt{d_{1}} \sin \left(\sqrt{d_{1}} \cdot t\right)
$$

the speed of the projectile at this moment.

From the accepted assumptions $v_{i}^{k}$ from the first law of thermodynamics, taking into account the PG adiabatic expansion and, accordingly, $I=\gamma \cdot U$, it follows:

$$
I_{\text {res }}=I_{\text {com }}-\gamma \cdot \varphi M \frac{\left(v_{i}^{k}\right)^{2}}{2} \text {. }
$$

Here $U$ - internal energy of the PG spent on work $A$ to accelerate the projectile with a fictitious mass $\varphi M$, $I_{\text {com }}$ - total enthalpy introduced from the PG into the projectile space, $I_{\text {res }}$ - residual enthalpy by the end of the current calculation step after the work on acceleration of the projectile has been completed. Equation (17) can be regarded as a simplified analogue in the enthalpy form of the Resal equation. From the ratio:

$$
I_{\text {res }}=\frac{\gamma}{\gamma-1} \cdot P_{i}^{k} \cdot W_{i}^{k} \Rightarrow P_{i}^{k}=\frac{\gamma-1}{\gamma} \cdot \frac{I_{r e s}}{W_{i}^{k}},
$$

the PG pressure is determined.

Based on the above expressions, it can be stated that a process has been identified that can provide the formation of free carbon in the PG during the movement of the projectile in the barrel. A model of this process has been developed, which is based on taking into account the change in the PG temperature along the length of the barrel.

The proposed model can serve as a basis for determining the conditions for the formation of a condensed phase in the form of free carbon (disproportionation of carbon monoxide - the Boudouard-Bell reaction), which makes it possible to obtain the boundaries of its formation along the length of the well.

5. 2. Method for determining the change in the temperature of the gunpowder gases along the length of the barrel in the firing process

The method for determining the change in PG temperature is based on the consideration of equilibrium processes occurring in an ideal gas. This is due to the fact that the covolum value is $\sim 0.001$. With the existing accuracy of specifying the initial data, not taking into account the covolum does not significantly affect the accuracy of the calculations. But, in turn, accounting for it complicates the calculations.

Accepted raw data:

- the mass of the first part of the burnt gunpowder is determined on the basis of the equation of state of an ideal gas from the condition of ensuring the selected forcing pressure in the free volume of the charging chamber. The rest of the gunpowder charge is divided into equal parts;

Accepted limitations and assumptions:

- PGs are a mixture of ideal gases, covolum is not taken into account;

- combustion rate of the gunpowder charge depends only on the pressure;

- volume of the gunpowder charge is not taken into account;

- free volume of the charging chamber does not change with the combustion of the gunpowder charge and is equal to the initial one. This is due to the fact that the volume of the projectile space is significantly greater than the volume of the gunpowder charge;

- PG pressure at all points in the space between the bottom of the charging chamber and the bottom of the projectile is the same (averaged);

- when calculating the subsequent finite element in the form of a mass of burnt gunpowder charge, an increase in the amount of PG in the space between the charging comora and the projectile occurs instantly;

- process of PG expansion is adiabatic, the adiabatic exponent is known;

- back pressure in front of the projectile is not taken into account;

- final elements of the incoming PG do not mix in the process of their expansion;

- at each step of the calculation, the number of elements taken into account increases by one, corresponding to the next portion of the burnt gunpowder charge;

- PG expansion time is determined at the next calculation step;

- energy costs for processes, in addition to accelerating the projectile, are taken into account using a coefficient that increases the mass of the projectile in the calculations (fictitious mass of the projectile).

The calculation method is based on a model presented in the form of a set of elements of PG portions (analogue of the finite element method) along the barrel length (one-dimensional formulation). The size of the element is determined by the fraction of the burnt gunpowder charge. For all elements, it is the same and is determined at the stage of selecting the initial data and ranged from $0.3 \mathrm{~kg}$ to $0.5 \mathrm{~kg}$. As the next fraction burns out, the formed PGs in the form of the next element enter the space behind the projectile. The PG pressure changes. The expansion of all elements of the PG that have entered the space behind the projectile by this time is considered. The calculated value of the PG pressure is used to determine the combustion time of the next portion of the gunpowder charge. During the same period of time, the expansion of the changed amount of PGs is considered.

The use of the modified model of the processes of PG expansion required a change in the form of writing its basic equations. The average temperature of the PG over the entire space behind the projectile made it possible to use the magnitude of the force of the gunpowder to take into account the energy introduced during the combustion of the gunpowder charge. Taking into account the change in temperature along the length of the space behind the projectile required taking into account the change in the energy of each PG element with their equal mass. This was achieved by writing an analogue of the Resal equation in enthalpy form for each finite element of the PG.

The following approach is proposed to describe the process of firing:

- movement of the projectile along the barrel is broadly divided into two parts: during the burning of gunpowder and after its end;

- gunpowder charge burns in increments of equal mass;

- pressure at which the next discrete of the charge is burned is considered constant and is taken to be equal to the pressure after the end of the PG expansion at the previous step of the calculation. This pressure value determines the combustion time of the next fraction of the charge $\left(t_{i}\right)$. It is the time interval for the next step. Depending on the current 
pressure, the combustion time interval for the next fraction of the charge and, accordingly, the calculation time interval at the next step will be variable;

- the formation of the next portion of PG of mass mi occurs without affecting the already existing PG (for example, outside the barrel). This step corresponds to the assumption about not taking into account the volume of the gunpowder charge and, therefore, not taking into account the change in the projectile volume as the gunpowder burns;

- the next generated portion of the PG enters the charging clod instantly through its end surface (Fig. 4). An instantaneous increase in PG pressure occurs and a new value $\left(p_{i}\right)$ is formed, which is determined by the amount of added PG, as well as the volume $\left.w_{0}, w_{1}, \ldots w_{i-2}, w_{i-1}\right)$ and the pressure of the existing PG;

- during the time interval $t_{i}$ (the duration of the next step of calculation), the PG expands and the projectile accelerates (excluding the PG inflow). At the end of the considered time interval, the value of the PG pressure is formed, which is used to determine the combustion time of the next fraction of the gunpowder charge at the next time step;

- after the combustion of the gunpowder charge, the calculation of the PG expansion and the acceleration of the projectile before it leaves the barrel is carried out.

At this stage, the distribution of the PG temperature along the barrel length is modeled during the firing process. In the calculated dependencies, the average values of some values are used, corresponding to modern barrel systems.

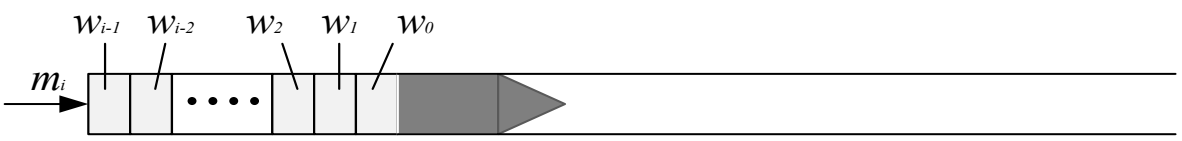

Fig. 4. Scheme of the formation of the incoming fraction of gunpowder gases at the next step of calculation

In accordance with the accepted assumptions, a method for solving a mathematical model is proposed.

Step 1. From (7)-(9), taking into account (6), the PG temperature is determined, and from the equation of state the amount of gunpowder charge required to form the boost pressure in the charging chamber. The value of the forcing pressure, the volume of the charging chamber (without taking into account the volume of the charge), and the adiabatic index are set. In accordance with the part of the burnt charge, the enthalpy of the considered PG volume is determined. The remaining mass of the gunpowder charge is determined, the number of calculation steps is selected. The mass of the part of the charge $\left(m_{i}\right)$, the combustion of which is considered at one calculation step, is determined. The masses of all parts are the same. The forcing pressure is the initial pressure for the next step of the calculation, it determines the combustion rate of a part of the gunpowder charge. In what follows, this PG volume is denoted as $w_{0}$ (Fig. 4).

Step 2. Using the final pressure from step 1 of their calculation (10), the burning time $t_{i}$ of part $m_{i}$ of the gunpowder charge is determined. According to the combustion law $u_{1}=u_{0} \cdot P$ and constant combustion area $F$.

Step 3 . The formed part of the PG is introduced (instantly) into the projectile space without changing its volume. In accordance with the current $i$-th number of the calculation step, this PG part will henceforth be denoted as $w_{i}$. The PG enthalpy $\left(I_{i}^{n}\right)$ is calculated as the sum of all enthalpy from the end of the previous step of calculation and introduced with the current part. In accordance with (11), the PG mixture pressure $\left(P_{i}^{n}\right)$ is determined at the beginning of the calculation step. Taking into account the assumption that the PG volumes from the corresponding calculation steps do not mix, their new values are determined.

Step 4. PG expansion and the projectile acceleration at the current calculation step occurs during the time $\mathrm{t}_{\mathrm{i}}$ determined at step 2 and is described by relation (12). The value $P$ is determined from relation (13), which describes the adiabatic process of gas expansion. Taking into account $W_{i}=S \cdot x_{i}$ and (13), expression (12) can be written in the form (14). The second term of equation (14) is linearized based on the Taylor series at the point $x_{i}^{n}$. Let's use the values of the quantities and the velocity of the projectile vin as the initial conditions at the initial moment of the calculation at the current step (the final speed at the previous step of the calculation). Assuming, as before, $\gamma=1.2$, solution (14) can be written in the form (15). Substituting the value of $t_{i}$ into this expression, the size of the projectile space by the end of the current calculation step is determined, and from (16) - the projectile velocity $v_{i}^{k}$ at this moment.

Step 5. For the accepted assumptions, from the first law of thermodynamics, taking into account the adiabatic expansion of the PG and, accordingly, $I=\gamma \cdot U$, by the end of the current calculation step, (17) follows. PG pressure at the end of the calculation step is determined from relation (18).

Step 6. If by the beginning of the next step of calculation not all of the gunpowder charge has been used, go to step 2; otherwise, further by the solution method.

Step 7. The calculation is carried out in accordance with the method described in paragraphs $2-5$, but without taking into account the entry into the projectile space of the PG. The end time of the calculation is determined by the moment the projectile reaches the muzzle.

During the calculation, for each element $w_{i}$ of the PG (Fig. 4), the current temperature can be determined from the equation of state.

Based on the outlined sequence of actions for the application of the expressions of the mathematical model, it can be stated that a computational method has been developed for determining the change in temperature and energy of gunpowder gases along the length of the barrel during the firing process. It is built on the basis of a modified model of the PG expansion, based on the finite element method with a constant mass. This made it possible to obtain a solution to the problem in a distributed setting.

5. 3. Results of modeling the temperature distribution in the space of the barrel between the charging ball and the moving projectile

After solving the model of the expansion of gunpowder gases using the proposed method for solving this model, results were obtained that characterize the temperature distributions of the gas in the process of a shot for the model system.

The initial data for modeling are given in Table 2.

The results are obtained for the averaged version of some variables. Their values are accepted and presented in Table 3, as reference (control) ones. The calculations are constructed in such a way as to ensure their values are close to those given in Table 3. 
Table 2

Shot parameters of the model artillery system

\begin{tabular}{|l|c|c|}
\hline \multicolumn{1}{|c|}{ Name } & Designation & Value \\
\hline Caliber & $d, \mathrm{~m}$ & 0.152 \\
\hline Sectional area of the barrel & $S, \mathrm{~m}^{2}$ & 0.0181 \\
\hline $\begin{array}{l}\text { Free volume of the charging chamber } \\
\text { (excluding the volume of gunpowder) }\end{array}$ & $W_{C C}, \mathrm{~m}^{3}$ & 0.0155 \\
\hline $\begin{array}{l}\text { Length of the free volume of the } \\
\text { charging box }\end{array}$ & $X_{C C}, \mathrm{~m}$ & 0.85 \\
\hline Projectile weight & $M, \mathrm{~kg}$ & 46 \\
\hline Gunpowder weight full/reduced charge & $m, \mathrm{~kg}$ & $18,4 / 11$ \\
\hline Corresponding muzzle velocity & $\mathrm{m} / \mathrm{s}$ & $945 / 775$ \\
\hline Power of gunpowder & $f, \mathrm{~J} / \mathrm{kg}$ & $0.95 \cdot 10^{6}$ \\
\hline Adiabatic exponent & $\Gamma$ & 1.2 \\
\hline $\begin{array}{l}\text { Upper temperature limit of the } \\
\text { Boudouard-Bell reaction }\end{array}$ & $K$ & 1300 \\
\hline Length of the rifled barrel & $\mathrm{M}$ & 6,185 \\
\hline Forcing pressure & $\mathrm{Pa}$ & $(3 \ldots 5) \cdot 10^{7}$ \\
\hline
\end{tabular}

Reference values

Table 3

\begin{tabular}{|l|c|c|}
\hline \multicolumn{1}{|c|}{ Name } & Designation & Value \\
\hline Forcing pressure & $P_{f}, \Pi \mathrm{a}$ & $3 \cdot 10^{7}$ \\
\hline Projectile speed (for reduced charge) & $V, \mathrm{~m} / \mathrm{s}$ & 775 \\
\hline $\begin{array}{l}\text { Projectile speed at the end of the burning } \\
\text { of gunpowder }(\tilde{V}=0.8 \cdot V)\end{array}$ & $\tilde{V}, \mathrm{~m} / \mathrm{s}$ & 620 \\
\hline
\end{tabular}

As a result, it was determined that when burning $0.5 \mathrm{~kg}$ of a charge with a stationary projectile, a pressure of $\sim 3.06 \cdot 10^{7} \mathrm{~Pa}$ will be reached, which is close to the accepted forcing pressure. The process of burning the remaining $10.5 \mathrm{~kg}$ of charge is divided into 35 steps according to $\Delta m=m_{i}=0.3 \mathrm{~kg}$. By choosing the value of the expression in parentheses in (10), the values $\tilde{V}=633.5 \mathrm{~m} / \mathrm{s}$ and $V=774.5 \mathrm{~m} / \mathrm{s}$ were obtained, which are close to the reference data (Table 3 ). In this case, $u_{0} \cdot F=8.65 \cdot 10^{-8} \mathrm{~m}^{3} / \mathrm{s}$ is taken.

The obtained reference values of the projectile velocities correspond to the tabular data for the considered charge with a known characteristic. The model includes the laws of conservation and the laws of chemical kinetics. The same laws are used in well-known lumped-parameter models. Models with distributed parameters for pressure are known, which are based on the same laws. This allows to assume that the temperature distribution obtained on the basis of the same laws adequately reflects the ongoing processes.

The graphs in Fig. 5-7 show the calculation results based on the data in Tables 2, 3. In (Fig. 5, $a$ ) the change in pressure is shown, and in (Fig. 5, $b$ ) - the temperature distribution in the projectile space at different positions of the projectile (Fig. 4).

The pressure in Fig. 6a is displayed for a point anchored to the bottom of the projectile. This explains the position of the beginning of the graph. On the abscissa axis, it is determined by the length of the charging chamber. The ordinate is the value of the forcing pressure. In Fig. 5, $b$, the temperature values are displayed for points tied to the corresponding parts of the PG. All parts have the same mass (in this case, $0.3 \mathrm{~kg}$ ). But at different pressures at the considered moment of time, they have different lengths. For this reason, the coordinates of the beginnings of graphs $1-3$ in Fig. $5, b$ on the abscissa axis do not coincide.
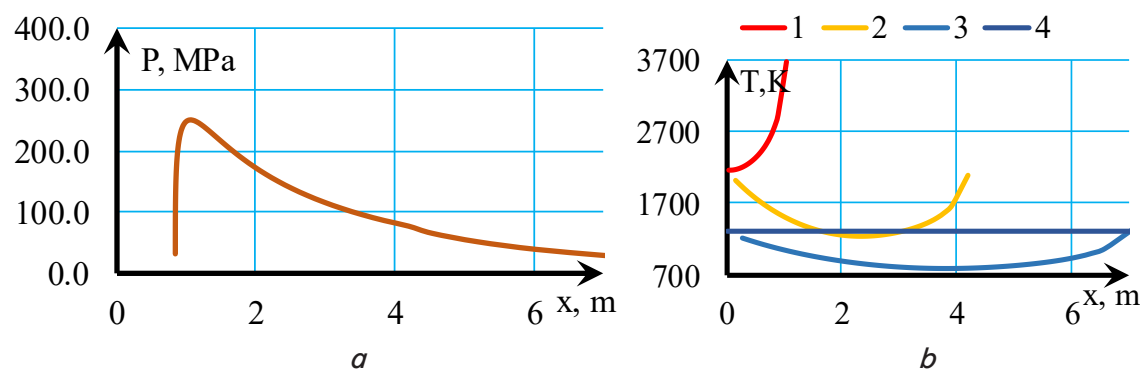

Fig. 5. The results of calculating the pressure and temperature of the gunpowder gases for a reduced charge of fresh gunpowder (based on the data in Tables 2, 3): $a$ - the change in pressure in the space behind the projectile; $b-\mathrm{PG}$ temperature distribution at various positions of the projectile: $1-$ at the moment of maximum pressure in the space behind the projectile; 2 - at the end of the combustion of the gunpowder charge; $3-$ when the projectile is at the muzzle; 4 - temperature limit of the Boudouard-Bell reaction
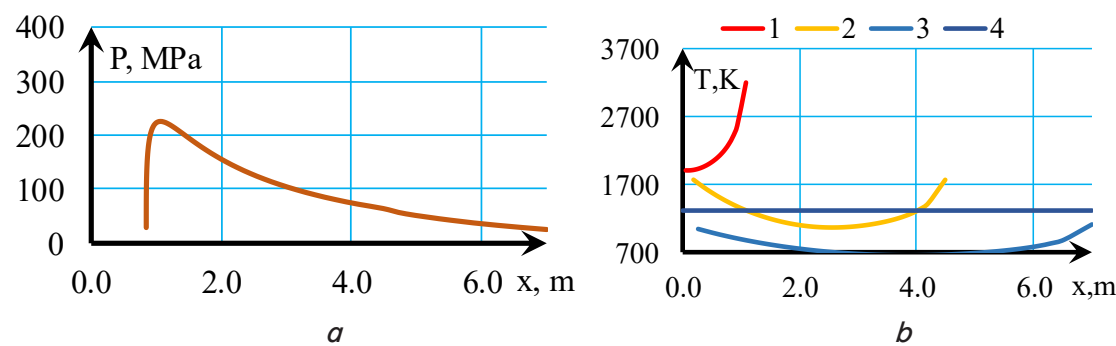

Fig. 6. The results of calculating the pressure and temperature of the gunpowder gases for a reduced charge of degraded gunpowder (based on the data in Tables 2, 3): $a$ - the change in pressure in the space behind the projectile; $b-\mathrm{PG}$ temperature distribution at various positions of the projectile: $1-$ at the moment of maximum pressure in the space behind the projectile; 2 - at the end of the combustion of the gunpowder charge; 3 - when the projectile is at the muzzle; 4 - temperature limit of the Bou Boudouard-Bell reaction 
To assess the effect of the degree of degradation of the gunpowder charge on the value of the parameters of the shot, a calculation was made for the gunpowder with the same gross formula (8) with a decrease in its energy capacity by $8 \%$ (7). For specific enthalpy $I=5240 \mathrm{~J} / \mathrm{kg}$ and other data corresponding to Tables 2, 3, the results shown in Fig. 7. Designations similar to Fig. 6 are used.

As a result, the characteristic velocities are determined as $\tilde{V}=618.5 \mathrm{~m} / \mathrm{s}$ and $V=750 \mathrm{~m} / \mathrm{s}$.

To estimate the range of the PG temperature at different charge values, a calculation was made for the mass of fresh gunpowder $m=18.4 \mathrm{~kg}$ (full charge). The calculation results are shown in Fig. 7. Designations similar to Fig. 6 are used.

The reference values for the case under consideration are the values of the velocities $V=945 \mathrm{~m} / \mathrm{s}$ and $\tilde{V}=756 \mathrm{~m} / \mathrm{s}$. As a result of the calculation, the values $V=944 \mathrm{~m} / \mathrm{s}$ and $\tilde{V}=780 \mathrm{~m} / \mathrm{s}$ were obtained.

Based on the data obtained on the distribution of the PG temperature along the barrel length (Fig. 6,7), the amount of free carbon that can be formed as a result of the Boudouard-Bell reaction was determined. The upper bound is fulfilled for two boundary cases. Considered: a reduced charge $(m=11 \mathrm{~kg})$ of degraded gunpowder $(I=5240 \mathrm{~J} / \mathrm{kg})$ and a full charge $(m=18.4 \mathrm{~kg})$ of fresh gunpowder $(I=5700 \mathrm{~J} / \mathrm{kg})$. The calculations were performed on the basis of solving problem (7)-(9) for determining the composition of PGs and the fraction of the formed free carbon depending on their temperature. Based on these data, the known masses of the parts of the PG (Fig. 4) and the calculated values of the temperatures of the parts of the PG (Fig. 6, 7), the amount of free carbon that can be released in each part of the PG was determined. The results are shown in Fig. 8, and for the case of a reduced charge of degraded gunpowder and in Fig. 8, $b$ for the case of a full charge of fresh gunpowder.

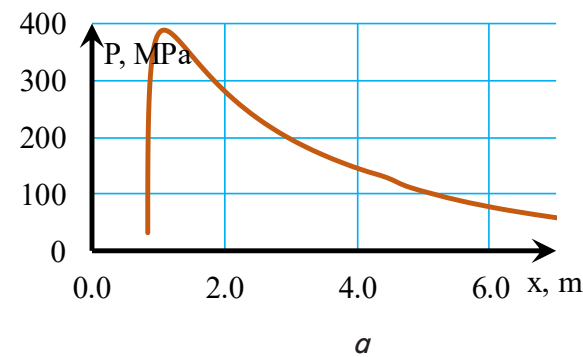

The simulation results showed that the boundaries of the formation of the condensed phase of free carbon along the length of the barrel during the shot were found, which was based on the obtained modified model of the expansion of the gunpowder gases and the method for its solution.

\section{Discussion of the results of the study of the temperature distribution in the space of the barrel between the charging chamber and the moving projectile}

The proposed modified model of internal ballistics processes is built using significant assumptions and serves for preliminary calculations. Its main task is to establish a connection between the parameters of the processes of internal ballistics and the phenomena of the external manifestation of a shot. The results obtained reflect the ambiguity of this relationship. So, in the case of using a reduced charge of fresh gunpowder until the end of the combustion of the gunpowder charge (Fig. 5, b: graphs 1,2), there are no conditions for the Boudouard-Bell reaction to occur. The PG temperature throughout the projectile space is above the boundary of its beginning (Fig. $5, b$ : graph 4). But at the stage of PG expansion after the end of the combustion of the charge (Fig. 5, $b$ : graph 3), there are conditions for the formation of a condensed phase in the form of free carbon. In this case, when the projectile passes the muzzle, the highest temperatures are observed at the bottom of the projectile and the bottom of the charging chamber. In the middle part of the PG, their temperature for the longest period of time is below the boundary of the disproportionation reaction of carbon monoxide. In this part, the condensed phase can form in large quantities.

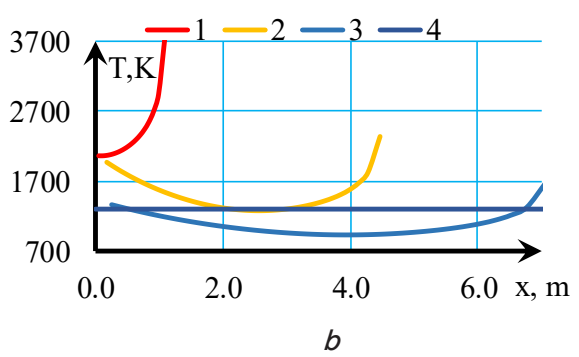

Fig. 7. The results of calculating the pressure and temperature of the gunpowder gases for a full charge of fresh gunpowder $(m=18.4 \mathrm{~kg}): a$ - pressure change in the space behind the projectile; $b-\mathrm{PG}$ temperature distribution at various positions of the projectile: 1 - at the moment of maximum pressure in the space behind the projectile; 2 - at the end of the combustion of the gunpowder charge; 3 - when the projectile is at the muzzle; 4 - temperature limit of the Boudoir-Bell reaction
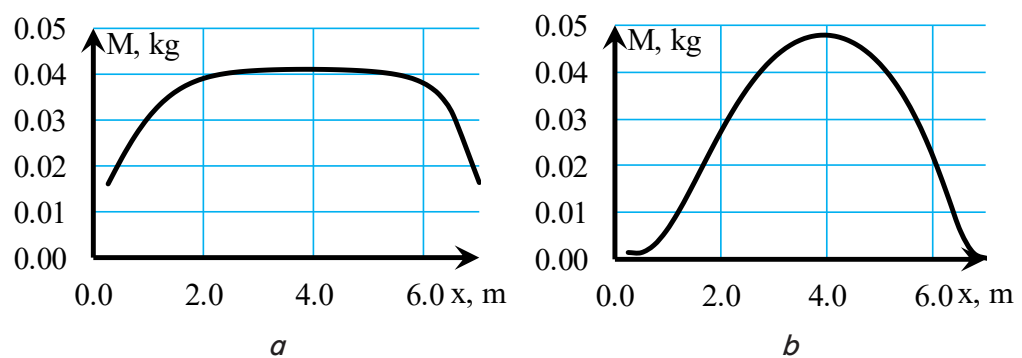

Fig. 8. Results of calculating the distribution along the length of the barrel of the mass of released free carbon in the corresponding PG part: $a$ - reduced charge of degraded gunpowder; $b$ - full charge of fresh gunpowder 
In the case of using a reduced charge of degraded gunpowder (Fig. 6), the nature of the change in the profile of the PG temperature remained similar to Fig. 5, $b$. But temperatures have undergone significant changes. In the case under consideration, the temperatures of most of the PGs and for a longer time are below the boundary that determines the possibility of the Boudouard-Bell reaction. Moreover, by the time the projectile reaches the muzzle, the PG temperature throughout the barrel becomes below the limit of the beginning of the formation of free carbon. This is manifested in its formation along the entire length of the barrel. This situation is reflected in the graph in Fig. 8, $a$ and corresponds to the photo of the external manifestation of the shot (Fig. 1,9), when free carbon occupies the entire length of the muzzle discharge.

In the case of using a full charge of fresh gunpowder (Fig. 7), a feature of the results obtained is the excess of the PG temperature at the bottom of the charging box and the muzzle cut of the boundary temperature for the Boudouard -Bell reaction. In other words, there should be no free carbon component in the initial and final parts of the muzzle discharge. This situation is reflected in the calculation results shown in the graph in Fig. 8, $b$ and they correspond to the external manifestation of the shot in the photo (Fig. 2). These photographs show areas with the absence of free carbon in the muzzle discharge in the head (Fig. 2, $b$ - red contour) and tail (Fig. 2, $c$ - yellow contour) parts of it.

Another feature of the muzzle discharge is the flash initiation point. So, from Fig. 1, $a, 2, a$ it follows that the initiation of the muzzle flash occurs in the frontal part of the cloud of free carbon (highlighted in red). At the same time, Fig. 9 indicates ignition from the point closest to the muzzle. In some cases, muzzle flash may not occur (Fig. 3).

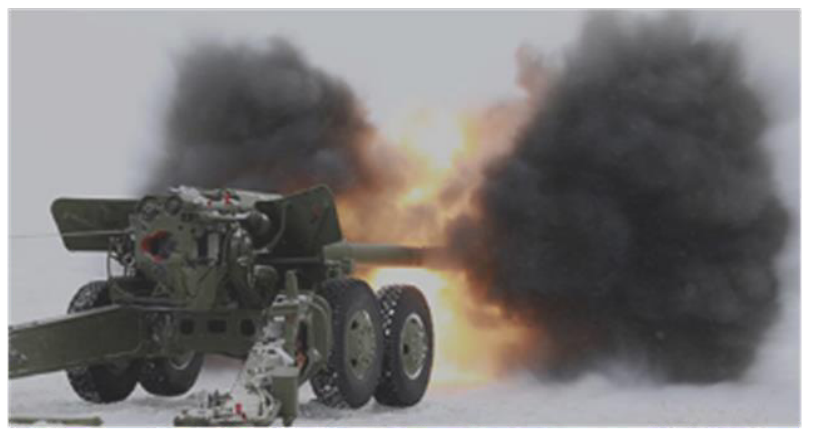

Fig. 9. A shot from a $152 \mathrm{~mm}$ cannon. Muzzle discharge flash from the muzzle brake side

The noted features of muzzle discharge can't be described on the basis of existing models of expansion of gunpowder gases. At the same time, the calculated results can serve as a basis for explaining the observed phenomena.

The flash initiation point can be determined on the basis of the type of profile of the PG temperature change along the barrel length: this is their maximum temperature in the area of the charging chamber and muzzle. In addition, the flash point of $\mathrm{H}_{2}(\sim 800 \mathrm{~K})$ and $\mathrm{CO}(\sim 900 \mathrm{~K})$ should be taken into account. The calculation results (Fig. 5-7) indicate the possibility of lowering the PG temperature below these limits. Their temperature continues to decrease even after leaving the barrel. As a result, there may be no muzzle flash (Fig. 3).
The features of the processes considered are manifested through the interconnection of the parameters of the charge, the parameters of the intraballistic processes and the external manifestations of the shot. The developed model of the PG expansion process can serve as a basis for determining the transfer function of the considered process of free carbon formation. It can provide an improvement to the known internal ballistics model. The difficulty lies in the fact that the parameters of this model can be determined only by solving the inverse problem. Namely: according to the amount of free carbon formed, the energy state (degree of degradation) of the gunpowder charge and the degree of wear of the barrel system are determined. The difficulties encountered in solving such problems are known.

One of the methods to overcome these difficulties is the library method [22]. Its feature is the preliminary solution of many direct problems with different initial data. This is done at the preparatory stage. And then, from the available list of solutions in real time, the desired parameters of the model and the characteristics of the charge are selected to solve the problem of internal ballistics.

The existence of an instability of the solution is possible due to the high sensitivity to the accuracy (change) of the transfer function parameters - the parameters of the model of internal ballistics processes. Taking into account the disproportionation reaction of carbon monoxide significantly affects the value of these parameters. So, in accordance with the results of calculations (Fig. 8, $a$ ) with a reduced charge $m=11 \mathrm{~kg}$, the mass of released free carbon can reach $\sim 1.3 \mathrm{~kg}$ or $11.8 \%$ of the initial mass of the charge. With a full charge $m=18.4 \mathrm{~kg}$ (Fig. $8, b$ ), the mass of released free carbon can reach $\sim 1.0 \mathrm{~kg}$ or $5.5 \%$ of the initial mass of the charge. Such a change in the amount of PGs leads to a significant decrease in the magnitude of their pressure. Failure to take into account such a phenomenon distorts the results of the solution.

In the future, based on the proposed model and the method for its solution, a new method can be built. Its characteristic feature may be the determination in real time in the process of firing the state of the charging chamber, the barrel barrel, the state of the gunpowder artillery system [23]. Basically, this presupposes the solution of the inverse problem of internal ballistics in combination with all the complexities of the corresponding computational process.

\section{Conclusions}

1. On the basis of the analysis of the conditions under which the PG expansion in the barrel of the barrel occurs, a chemical reaction has been determined, which makes it possible to explain the formation of free carbon during the firing process. It describes the process of disproportionation of carbon monoxide (Boudouard-Bell reaction). Its feature is the shift of the equilibrium point towards the formation of free carbon as the temperature decreases. A special condition is the need to sharply reduce it. It is these conditions that are realized in the process of firing. The mathematical model of the PG expansion process was further developed. The PG model is presented in the form of the PG gross formula and their molar mass. The specific enthalpy of the gunpowder charge is determined by the strength of the gunpowder taking into account the constant adiabat. The molar mass 
of PG is determined on the basis of the results of solving the problem of chemical kinetics of combustion of gunpowder. The modeling of the parameters of the PG expansion process is based on the equations of the first law of thermodynamics for the adiabatic process and Newton's second law in differential form. The proposed model made it possible to determine the temperature change along the length of the barrel. This made it possible to determine the conditions for the possible formation of a condensed phase in the form of free carbon (disproportionation of carbon monoxide Boudouard-Bell reaction).

2. A computational method has been developed for determining the change in temperature and energy of gunpowder gases along the length of the barrel in the process of firing on the basis of a modified model of the PG expansion process. The method is based on an approach similar to the finite element method. Its feature is the use of a part of the gunpowder charge as a characteristic mass value. Another feature is the variable number of finite elements in the process of solving the model problem of the combustion of gunpowder and the PG formation. The use of this approach made it possible to obtain a solution for the distributed formulation based on the sequential solution of a number of lumped problems.

3. The boundaries of the process of formation of the condensed phase in the form of free carbon along the length of the barrel in the process of firing were found. The results were obtained on the basis of a modified model of the expansion of gunpowder gases and a method for its solution. The distribution of the PG temperature in the space of the barrel between the charging ball and the moving projectile has been determined. On the basis of these data, changes in the length of the zone of the Boudouard-Bell reaction (the zone of formation of free carbon) were determined. The results are obtained for the charge of fresh and degraded gunpowder, for cases of full and reduced charges. For the indicated initial data used as boundary, the amount of liberated free carbon along the length of the barrel was determined as an upper estimate. This made it possible to explain the features of the distribution of free carbon in muzzle emissions. Thus, a connection has been identified and a model has been proposed that describes the influence of the initial data on the external manifestation of the shot. Analysis of the simulation results showed the reason for the possibility of initiating a secondary flash of the muzzle discharge both from the front side and from the side of the muzzle brake. The assessment of the total amount of released free carbon showed a significant effect of the disproportionation reaction of carbon monoxide on the processes of internal ballistics and the need to take it into account in the models of the firing process.

\section{References}

1. Serebriakov, M. E. (1962). Vnutrenniaia ballistika stvolnykh sistem i porokhovykh raket. Moscow, 702.

2. Carlucci, D. E., Jacobson, S. S. (2008). Ballistics: theory and design of guns and ammunition. Taylor \& Francis Group, 502.

3. Rashad, M. M., Zhang, X. B., Elsadek, H. (2013). Numerical simulation of interior ballistics for large caliber guided projectile naval gun. Journal of Engineering and Applied Science, 60 (2), 163-176. Available at: https://www.researchgate.net/ publication/264786882

4. Jang, J.-S., Oh, S.-H., Roh, T.-S. (2016). Development of three-dimensional numerical model for combustion-flow in interior ballistics. Journal of Mechanical Science and Technology, 30 (4), 1631-1637. doi: http://doi.org/10.1007/s12206-016-0319-y

5. Rusiak, I. G., Ushakov, V. M. (2001). Vnutrikamernye geterogennye protsessy v stvolnykh sistemakh. Ekaterinburg: UrO RAN, 259.

6. Li, P., Zhang, X. (2021). Numerical research on adverse effect of muzzle flow formed by muzzle brake considering secondary combustion. Defence Technology, 17 (4), 1178-1189. doi: http://doi.org/10.1016/j.dt.2020.06.019

7. Steward, B. J., Perram, G. P., Gross, K. C. (2011). Visible and Near-Infrared Spectra of the Secondary Combustion of a $152 \mathrm{~mm}$ Howitzer. Applied Spectroscopy, 65 (12), 1363-1371. doi: http://doi.org/10.1366/11-06445

8. Steward, B. J., Bauer, K. W., Perram, G. P. (2012). Remote discrimination of large-caliber gun firing signatures. Journal of Applied Remote Sensing, 6 (1), 063607. doi: http://doi.org/10.1117/1.jrs.6.063607

9. Steward, B. J., Gross, K. C., Perram, G. P. (2011). Reduction of optically observed artillery blast wave trajectories using low dimensionality models. Airborne Intelligence, Surveillance, Reconnaissance (ISR) Systems and Applications VIII. doi: http://doi.org/ $10.1117 / 12.883524$

10. Zakharenkov, V. F. (2010). Vnutrenniaia ballistika i avtomatizatsiia proektirovaniia artilleriiskikh orudii. Saint Petersburg, 276. Available at: https://ua1lib.org/book/3064917/757a40?id=3064917\&secret $=757 \mathrm{a} 40$

11. Li, X., Mu, L., Zang, Y., Qin, Q. (2020). Study on performance degradation and failure analysis of machine gun barrel. Defence Technology, 16 (2), 362-373. doi: http://doi.org/10.1016/j.dt.2019.05.008

12. Kriukov, O., Melnikov, R., Bilenko, O., Zozulia, A., Herasimov, S., Borysenko, M. et. al. (2019). Modeling of the process of the shot based on the numerical solution of the equations of internal ballistics. Eastern-European Journal of Enterprise Technologies, 1 (5 (97)), 40-46. doi: http://doi.org/10.15587/1729-4061.2019.155357

13. Alemasov, V. E., Glushko, V. (1974-1976). Thermodynamic and thermophysical properties of combustion products. Jerusalem: Israel Program for Scientific Translations. Available at: https://searchworks.stanford.edu/view/892711

14. Brunetkin, O., Maksymov, M. V., Maksymenko, A., Maksymov, M. M. (2019). Development of the unified model for identification of composition of products from incineration, gasification, and slow pyrolysis. Eastern-European Journal of Enterprise Technologies, 4 (6 (100)), 25-31. doi: http://doi.org/10.15587/1729-4061.2019.176422 
15. Rout, K. R., Gil, M. V., Chen, D. (2019). Highly selective CO removal by sorption enhanced Boudouard reaction for hydrogen production. Catalysis Science \& Technology, 9 (15), 4100-4107. doi: http://doi.org/10.1039/c9cy00851a

16. Krylova, A. Y. (2014). Products of the Fischer-Tropsch synthesis (A Review). Solid Fuel Chemistry, 48 (1), 22-35. doi: http://doi.org/ 10.3103/s0361521914010030

17. Kogler, M., Köck, E.-M., Klötzer, B., Schachinger, T., Wallisch, W., Henn, R. et. al. (2016). High-Temperature Carbon Deposition on Oxide Surfaces by CO Disproportionation. The Journal of Physical Chemistry C, 120 (3), 1795-1807. doi: http://doi.org/10.1021/ acs.jpcc.5b12210

18. Mianowski, A., Robak, Z., Tomaszewicz, M., Stelmach, S. (2012). The Boudouard - Bell reaction analysis under high pressure conditions. Journal of Thermal Analysis and Calorimetry, 110 (1), 93-102. doi: http://doi.org/10.1007/s10973-012-2334-2

19. Burnham, A. K., Fried, L. E. (2006). Kinetics of PBX9404 aging. UCRL-CONF-224391. 7th aging, compatibilityand stockpile stewardship conference. Los Alamos, 6. Available at: https://www.osti.gov/biblio/894349-kinetics-pbx9404-aging

20. Anipko, O. B., Khaikov, V. L. (2012). Methods analysis for assessment of propellant charges as a part of the artillery ammunition monitoring system. Integrirovannye tekhnologii i energosberezhenie, 3, 60-71. Available at: http://repository.kpi.kharkov.ua/ handle/KhPI-Press/2199

21. Brunetkin, O., Davydov, V., Butenko, O., Lysiuk, G., Bondarenko, A. (2019). Determining the composition of burned gas using the method of constraints as a problem of model interpretation. Eastern-European Journal of Enterprise Technologies, 3 (6 (99)), 22-30. doi: http://doi.org/10.15587/1729-4061.2019.169219

22. Pelykh, S. N., Maksimov, M. V., Baskakov, V. E. (2008). Model of cladding failure estimation under multiple cyclic reactor power changes. The 2-nd International Conference 'Current Problems in Nuclear Physics and Atomic Energy', 638-641. Available at: https://inis.iaea.org/search/search.aspx?orig_q=RN:40062726 